\title{
Neurofibromatosis - Neurosurgical Treatment and Follow-up
}

\author{
a report by \\ Madjid Samii and Venelin M Gerganov
}

International Neuroscience Institute, Otto-von-Guericke University, Hannover

\section{Neurofibromatosis Type I}

Neurofibromatosis type I (NFI), also known as von Recklinghausen disease, is one of the most common inherited diseases in humans. Its incidence is one per 3,500-4,000 live births and it affects both sexes equally. NFI is an autosomal-dominant disorder and is the result of a mutation of a gene mapped to chromosome 17, which is the NFI gene. ${ }^{1,2}$ The product of the gene is a protein called neurofibromin, a GTPase-activating protein (GAP) that helps to maintain the protooncogene Ras in an inactive form. NFI is characterised by 100\% penetrance but varying expressivity.

The diagnostic criteria for NFI have been formulated by the National Institutes of Health $(\mathrm{NIH})$ Consensus Development Conference on $\mathrm{NF}^{3}{ }^{3}$ and include six or more café-au-lait macules (CALM), two or more neurofibromas of any type or one or more pleximorm neurofibroma, two or more Lisch nodules, distinct osseous lesions and a first-degree relative with NFI. If two or more of these signs are present, the diagnosis is NFI. Although direct sequencing could be used to detect the causative mutation for individuals who meet these diagnostic criteria for $\mathrm{NFI}$, molecular testing is generally not necessary.

Patients with NFI manifest clinically with cutaneous, ophthalmological, musculoskeletal and/or neurological symptoms. 4,5 The cutaneous and ocular manifestations are the most common and typically are CALM, axillary freckling and Lisch nodules. CALM are apparent in 99\% of patients and are usually present at birth. Musculoskeletal abnormalities include bone abnormalities, such as skeletal dysplasia (particularly

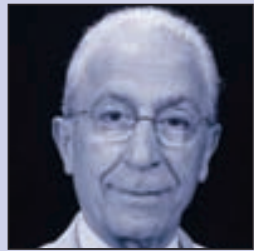

Madjid Samii is President of the International Neuroscience Institute at Otto-von-Guericke-University. He was Director of the Neurosurgical Clinic at Nordstadt Hospital, Hannover, from 1977 to 2002 and Chair of Neurosurgery at the Medical School of Hannover from 1988. In 1992, he was elected as President of the International Skull Base Society (ISBS) and President of the World Federation of Skull Base Societies. He also served as President of the German Society of Neurosurgery from 1996 to 1998. Dr Samii is Editor of 15 neurosurgical books and more than 500 publications on peripheral nerves, spinal tumours and syringomyelia, skull base pathology, brain tumours and cranial nerve disorders. He has given more than 1,000 lectures as invited and/or honoured guest of numerous national and international congresses.

E: samii@ini-hannover.de

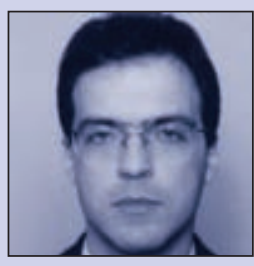

Venelin M Gerganov is a Neurosurgeon at the International Neuroscience Institute at Otto-von-Guericke University, Hannover, Germany. His research interests include skull base surgery, endoscopy, intra-operative and functional imaging and brain tumour biology and treatment. He received his medical and neurosurgerical training at the Pirogov Institute and Sofia Medical University, Bulgaria. sphenoid wing dysplasia), scoliosis and tibial pseudarthrosis. The neurological manifestations of $\mathrm{NFI}$ are variable and may be due to brain, spine or peripheral nerve tumours, epilepsy, macrocephaly, hydrocephalus, meningoceles and/or peripheral neuropathy

Neurofibromas in NFI manifest as cutaneous neurofibromas, subcutaneous neurofibromas, nodular plexiform neurofibromas and diffuse plexiform neurofibromas. ${ }^{6}$ In contrast with cutaneous neurofibromas, plexiform neurofibromas may undergo malignant transformation. Both NFI and NFIl patients are at increased risk of developing intra-cranial tumours; however, the tumour types are quite different. Brain tumours that have a higher incidence in NFI patients than in the general population are gliomas, ependymosmas, meningiomas and primitive neuroectodermal tumours (PNETs). Gliomas are typically lowgrade and involve the optic pathways, hypothalamus, cerebellum, brainstem and spinal cord.

Optic pathway tumours usually involve the anterior visual pathways and occur in $11-19 \%$ of patients. Almost half are asymptomatic at diagnosis, while in the others the symptoms are related to the location of the tumours along the optic pathways. The initial management of optic pathway gliomas is follow-up with serial neuroimaging and ophthalmological examinations. These tumours may have a more indolent natural history and are less aggressive than optic gliomas in patients without NFI. 4,5,7 Tumours involving the intra-orbital segment of the optic nerve cause progressive proptosis, papilledema and optic atrophy. Those involving the chiasm may extend to the hypothalamus and third ventricle and lead to severe endocrine disturbances and/or hydrocephalus. In cases of significant proptosis or visual loss, and particularly for unilateral tumours located anterior to the optic chiasm, surgical removal is an option. Residual tumour or regrowth after incomplete removal may be treated with chemotherapy (in children $<5$ years) or radiotherapy.

NFI patients may present with vascular complications. Strokes occur in approximately $1 \%$ of patients and the most common cause is the occlusion of the carotid or middle cerebral artery. ${ }^{1} \mathrm{NFI}$ has a significant effect on learning and cognition. Areas of signal hyperintensity on transverse relaxation (T2)-weighted magnetic resonance images (MRIs) the 'unidentified bright objects' - are observed in $60-70 \%$ of children with $\mathrm{NFI}$, but the relationship between unidentified bright objects and cognitive dysfunction remains controversial. Psychiatric disorders occur in $33 \%$ of patients, which is a much higher frequency compared with the general population.

The tailored therapeutic approach may provide a breakthrough in the management of NFI patients. In recent years, the identification of specific biochemical pathways in NFI has allowed the development of 
therapy targeted at blocking the precise dysfunctional pathway; this is being applied for the treatment of multiple tumour types. An example of this is the inactivation of Ras using farnesyl transferase inhibitors, which inhibit the post-translational modification and activation of Ras..$^{2,8}$

The introduction of more precise predictive tests, the further development of biologically based therapies and the team approach to their management will definitely lead to significant advances in the supervision of patients with NFI.

\section{The tailored therapeutic approach may provide a breakthrough in the management of neurofibromatosis type I patients.}

\section{Neurofibromatosis Type II}

NFII is an autosomal-dominant inherited disease with an incidence of approximately $1: 33,000$ to $1: 50,000.5,9$ The lifelong tendency to form new central nervous system tumours such as schwannomas, meningiomas, gliomas and neuromas pre-determines the impossibility of a definitive cure for these patients. Treatment is focused on life prolongation, preservation of cranial nerve function or auditory rehabilitation and, thus, the maintenance of quality of life. ${ }^{10}$ Management of bilateral vestibular schwannomas (VS) differs in a number of ways from sporadic unilateral tumours, and the major management concern is the disabling consequences of acquired deafness.

The criteria for NFII are bilateral VS or a parent, sibling or child with NFII, and either unilateral vestibulocochlear nerve tumour or any one of the following: neurofibroma, meningioma, glioma, schwannoma, posterior capsular cataract or opacity at a young age.11,12 NFIl is caused by a single germline mutation of the chromosome band $22 q 12 . .^{13}$ The normal allele is lost due to a somatic mutation in the cells giving rise to the tumour. It has been estimated that NFII has the highest spontaneous mutation rate of any human genetic disorder (approximately 50\%). If a person inherits the abnormal gene, there is a $95 \%$ chance that she or he will develop a bilateral VS. However, no evidence of other affected family members can be found in approximately $50 \%$ of patients. It is supposed that the disease is a sequence of two germline mutations (the 'double hit' hypothesis). No differences have been reported between mutations detected in patients with NFII and those in patients with sporadic tumours.

Molecular analysis of NFIl revealed that the mutation affects a gene that encodes a protein with 595 amino acids, otherwise known as the Schwannomin/Merlin protein. This gene product is thought to signal cellular growth inhibition and is related to a family of proteins - the ezrin-radixin-moesin family - that links the actin cytoskeleton to the cell membrane molecules. ${ }^{13-15}$

Bilateral VS that become manifest in the second or third decade of life are the hallmark of NFII and occur in about $95 \%$ of adult patients with
NFII. Children with NFIl often present with non-vestibular tumours. Clinical management of NFIl patients cannot be based on the expectation of similar natural evolution. ${ }^{11}$ Although some studies of the growth rate of VS in NFII indicate that it is generally higher in younger patients, there are great variations, both between patients and over time in the same patient. No predictors for the rate of increase of the tumours have been identified.

All of these characteristics, as well as the association with other central nervous system tumours, determine the scope of the problems facing the neurosurgeon. Treatment options include observation, radiosurgery or surgical treatment. ${ }^{9}$ Several surgical approaches have been put forward, such as partial or total tumour removal via the retrosigmoid or the middle fossa approach, aiming at hearing preservation, total removal of the tumour with placement of an auditory brainstem implant and decompression of the cochlear nerve at the internal auditory canal (IAC).

\section{Personal Series}

Over a period of more than 35 years, the senior author (MS) has operated on more than 165 patients with NFII. The total number of VS surgeries in these patients is 210 . Twenty-four per cent of all patients were deaf pre-operatively, with preserved unilateral hearing in $34 \%$ and with preserved bilateral hearing $42 \%$. Total tumour removal was achieved in $85 \%$ of the operated tumours. In $15 \%$, deliberate subtotal removal was performed for brainstem decompression and hearing preservation in the hearing ear only. The subtotal removal and IAC decompression led to long-term hearing preservation in $10.4 \%$ of the patients. The overall rate of hearing preservation in the series was $35 \%$. If patients with preserved useful pre-operative hearing only are included, the rate is $65 \%$. Twenty-three per cent of patients retained bilateral hearing after surgery. The anatomical integrity of the facial nerve was preserved in $89 \%$.

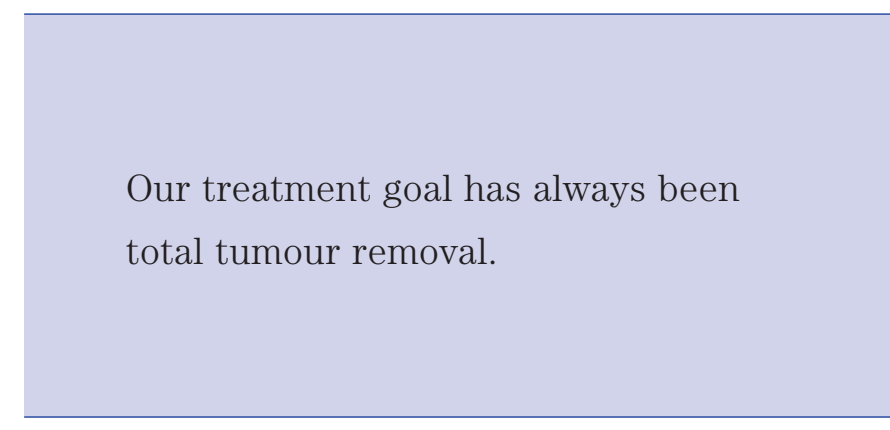

\section{Facial Nerve Preservation}

Some surgeons advocate subtotal tumour removal considering the absence of clear arachnoidal plane to the facial nerve and, correspondingly, the increased risk of its injury. However, regrowth of the residual tumour is highly probable because of the typical young age of the patients and the fact that in NFII VS characteristically grow rapidly. Surgeries in cases of recurrences are more difficult and more dangerous. Our treatment goal has always been total tumour removal. The only exceptions have been made in order to preserve hearing function or facial nerve integrity. Deliberate subtotal resections have been performed for brainstem decompression and for hearing preservation in the remaining hearing ear. Facial nerve preservation was possible in all cases except if the schwannoma (or multiple schwannomas) arise from the facial nerve, or no cleavage plane 
between the cranial nerves and the tumour could be found. In 187 cases (89\%), the facial nerve could be separated from the VS and its continuity preserved.

\section{Hearing Preservation}

A more conservative approach has been put forward in order to preserve hearing, i.e. simple observation of the patient and subtotal intracapsular resection if the VS increases. ${ }^{12}$ Some surgeons advise that surgery should be postponed for as long as possible. Furthermore, hearing conservation attempts should be performed on the side with the larger tumour, and surgery on the better hearing ear is to be avoided. If this treatment strategy is followed, surgery is usually performed when the tumours have reached a considerable size, which significantly worsens the outcome. We accept an initial observational period in selected cases, such as the elderly, those with poor surgical risks or those who refuse surgery.

\section{Our treatment philosophy is based on the assumption that surgical removal of vestibular schwannomas can preserve hearing.}

Proponents of the active treatment strategy state that surgical removal of bilateral VS should occur as early as possible if the tumour is small (up to $1.5 \mathrm{~cm}$ ), the hearing is usable and hearing preservation is possible. 10,16,18 Our treatment philosophy is based on the assumption that surgical removal of VS can preserve hearing. Our goal has always been the preservation of functional hearing for as long as possible. If the chances of functional hearing preservation are realistic, our recommendation is for early surgery. Tumour extension, audiometry data and auditory brainstem responses (ABR) determine which side should be operated on initially. The side with the best chance for hearing preservation is treated first.

The main predictors of successful hearing preservation are tumour extension, pre-operative hearing level and the quality of $A B R$. We recommend initial treatment on the side with the smaller tumour or the side with the better hearing level. If the hearing and the tumour size are similar on both sides, our decision is based on the pre-operative ABR. The side with better quality is operated on first, because the chances of successful hearing preservation are better on that side. Thus, we achieved bilateral hearing preservation in $23 \%$ of the patients, and preserved unilateral hearing after surgery in $65 \%$ of the patients, who had a useful pre-operative hearing level. For VS on the only hearing side we offer IAC decompression and complete or partial tumour removal, depending on the intra-operative ABR. If slight microsurgical actions are followed by severe deterioration in $A B R$, only partial resection is performed. With this strategy we succeeded in preserving hearing in 15 patients. Follow-up examinations indicated that preserved hearing remained functional for periods of up to 15 years. Tumour regrowth has been moderate and has not necessitated re-operations.

Radiosurgery is another treatment option. It provides tumour control in up to $81 \%$ of patients at 10 years and hearing preservation of approximately $33-43 \%$, although some deterioration occurs during the ensuing six years. ${ }^{18}$ In our opinion, radiosurgery is not the optimal primary therapy in NFII. It is best reserved for NFIl patients who have particularly aggressive tumours, those with medical contraindications for microsurgery, patients who refuse surgery or the elderly. ${ }^{10,11,16}$

Bilateral deafness of NFII patients is often inevitable. The introduction of the auditory brainstem implant in the clinical practice offers hope for such patients. ${ }^{19} \mathrm{~A}$ recently introduced promising alternative to auditory brainstem implants is the auditory mid-brain implant. 10,20

All patients with NFII - and their families - should have access to genetic testing because early, preferably pre-symptomatic, diagnosis improves clinical outcome. Some authors even suggest that MR tomography scanning for members of NFIl families should start at 10-12 years of age. ${ }^{15}$

Treatment of NFIl patients should be individualised and performed in specialised treatment centres. Close collaboration with the patient and his or her family is essential. The attitude and expectations of patients should guide the decision-making process. Carefully individualised treatment strategies offer the possibility of prolongation of life and preservation of neurological functions.
1. Lee MJ, Stephenson DA, Recent developments in neurofibromatosis type 1, Curr Opin Neurol, 2007;20:135-41.

2. Ward BA, Gutmann DH, Neurofibromatosis 1: from lab bench to clinic, Pediatr Neurol, 2005:32:221-8.

3. Neurofibromatosis, Conference Statement, National Institutes of Health Consensus Development Conference, Arch Neurol, 1988; 45:575-8.

4. Al-Otibi M, Rutka JT, Neurosurgical implications of neurofibromatosis Type I in children, Neurosurg Focus, 2006;20(1):E2.

5. Yohay K, Neurofibromatosis types 1 and 2, Neurologist, 2006; 12:8693.

6. Leonard JR, Ferner RE, Thomas N, Gutmann DH, Cervical cord compression from plexiform neurofibromas in neurofibromatosis 1, J Neurol Neurosurg Psychiatry, 2007;78(12):1404-6.

7. Thiagalingam S, Flaherty M, Billson $F$, et al., Neurofibromatosis type 1 and optic pathway gliomas: follow-up of 54 patients, Ophthalmology, 2004;111:568-77.

8. Packer RJ, Gutmann DH, Rubenstein A, et al., Plexiform neurofibromas in NF1: Toward biologic based therapy, Neurology, 2002;58:1461-70.

9. Neff BA, Welling DB: Current concepts in the evaluation and treatment of neurofibromatosis type II. Otolaryngol Clin North Am, 2005;38:671-84.

10. Samii M, Matthies C, Tatagiba M, Management of vestibular schwannomas (acoustic neuromas): auditory and facial nerve function after resection of 120 vestibular schwannomas in patients with neurofibromatosis 2, Neurosurgery, 1997;40: 696-706.

11. Baser ME, Gareth DR, Evans DG, Gutmann DH, Neurofibromatosis 2, Curr Opin Neurol, 2003;16:27-33.

12. National Institutes of Health Consensus Development Conference: Statement on Acoustic Neuroma, Arch Neurol, 1994;51:201-7.

13. Meng JJ, Lowrie DJ, Sun H, et al., Interaction between two isoforms of the NF2 tumor suppressor protein, merlin, and between merlin and ezrin, suggests modulation of ERM proteins by merlin, J Neurosci Res, 2000;62:491-502.

14. Bradley WD, Clinical manifestations of mutations in the neurofibromatosis Type 2 gene in vestibular schwannomas (acoustic neuromas), Laryngoscope, 1998;108:178-89.

15. Evans DG, Baser ME, O'Reilly B, et al., Management of the patient and family with neurofibromatosis 2: a consensus conference statement, Br J Neurosur, 2005:19:5-12.

16. Brackmann DE, Fayad JN, Slattery WH III, et al., Early proactive management of vestibular schwannomas in neurofibromatosis type 2, Neurosurg, 2001:49:274-80.

17. Slattery WH III, Brackmann DE, Hitselberger W, Hearing preservation in neurofibromatosis type 2, Am J Otol, 1998;18: 638-43.

18. Mathieu D, Kondziolka D, Flickinger JC, et al., Stereotactic radiosurgery for vestibular schwannomas in patients with neurofibromatosis type 2: an analysis of tumor control, complications, and hearing preservation rates, Neurosurgery, 2007:60:460-68

19. Lenarz T, Moshrefi M, Matthies $C$, et al., Auditory brainstem implant: part I. Auditory performance and its evolution over time, Otol Neurotol, 2001;22:823-33.

20. Lim $H$, Lenarz $T$, Joseph $G$, et al., Electrical stimulation of the midbrain for hearing restoration: Insight into the functional organization of the human central auditory system, J Neurosci, 2007;27(49):13541-51. 